\title{
Expanding spectrum of SCN1A-related phenotype with novel mutations
}

\author{
Semra Hız-Kurul ${ }^{1}$, Semra Gürsoy ${ }^{2}$, Müge Ayanoğlu${ }^{1}$, Uluç Yiş ${ }^{1}$, Derya Erçal ${ }^{2}$ \\ Divisions of ${ }^{1}$ Pediatric Neurology and ${ }^{2}$ Pediatric Genetics, Department of Pediatrics, Dokuz Eylül University Faculty of \\ Medicine, Izmir, Turkey. E-mail: dr.semra@hotmail.com \\ Received: 28th March 2017, Accepted: 1st May 2017
}

\begin{abstract}
SUMMARY: Hız-Kurul S, Gürsoy S, Ayanoğlu M, Yiş U, Erçal D. Expanding spectrum of SCN1A-related phenotype with novel mutations. Turk J Pediatr 2017; 59: 570-575.

Mutations in the genes encoding voltage-gated sodium channels cause a variety of epilepsy syndromes, with most of the mutations occurring in SCN1A gene. It is one of the most well-researched epilepsy genes. The SCN1A gene, which seems to be a relevant regulator of excitability of the CNS, is implicated in various epilepsy phenotypes through various genetic mechanisms ranging from common variants to rare monogenic variants. It is known that SCN1A gene is tightly linked to severe myoclonic epilepsy of infancy (SMEI). However, its phenotypic spectrum is expanding. Here, we report clinical and genetic findings of 10 patients with SCN1A mutations where two of them were found to have novel mutations. Our findings support understanding and updating knowledge on the correlation between phenotype distribution and location and type of mutations in SCN1A-related disorders.
\end{abstract}

Key words: SCN1A-related disorders, refractory epilepsy, severe myoclonic epilepsy of infancy.

Variants in SCN1A gene (MIM\#182389) were first reported in association with epilepsy in $2000 .{ }^{1}$ To date, more than 1,270 point mutations in the coding regions and more than 600 sequence variants including chromosomal microdeletions/duplications of the SCN1A gene have been identified. These mutations alter the electrophysiologic properties or disrupt the expression of sodium channel $\mathrm{Na}_{\mathrm{v}} 1.1, \alpha 1$. It is known that SCN1A mutations are most notably linked to two epilepsy syndromes, severe myoclonic epilepsy of infancy (SMEI), also known as Dravet syndrome (DS; MIM\#607208) and the mild familial epilepsy syndrome of genetic epilepsy with febrile seizures (FS) plus (GEFS+; MIM\#604233). ${ }^{1-4}$ All of the SCN1A mutations are dominantly inherited and they can result most commonly as observed in either loss of function in DS or altered function in GEFS +. DS is one of the most common and well-defined epileptic encephalopathy. It typically presents in infancy with prolonged, febrile and afebrile seizures. Myoclonic, focal, atypical absence and atonic seizures appear between the ages of 1 and 4 years. The epilepsy is usually not responsive to standard antiepileptic drugs (AEDs) and affected children develop an epileptic encephalopathy with cognitive, behavioral, and motor impairment.5,6 About $80 \%$ of DS patients have SCN1A mutation, and approximately $90 \%$ of these mutations arise de novo. In addition to individuals with SMEI whose features fulfill the diagnostic guideline for core SMEI, a group of patients have clinical features that are almost identical to those of core SMEI, but these are not necessarily fully consistent with the accepted diagnostic criteria. This entity is defined as SMEI-borderline (SMEB). It is not obvious whether SMEB is a phenotypic variant of SMEI and results from the same genetic etiology. 7,8

GEFS+ is a milder autosomal dominant familial epilepsy syndrome. The core phenotype in GEFS + families are "febrile seizures plus". It refers to FS persisting beyond the age of 6 years or FS in conjunction with other afebrile seizure types. In rare cases, GEFS+ phenotypes have been found to overlap with DS within a family. The phenotypes of SCN1A in GEFS+ are obviously variable, but the source of this 
variability is currently unknown.1,9,10

More recent studies have also indicated that common variants in the SCN1A gene may be associated with a wide range of common epilepsy phenotypes, including temporal lobe epilepsy, idiopathic/genetic generalized epilepsy (GGE/IGE), intractable childhood epilepsy with generalized tonic-clonic seizures, and less frequently and simple febrile seizures. ${ }^{11-13}$

In spite of the identification of many mutations in the SCN1A gene, the genotype-phenotype correlation is still unclear and the phenotypic spectrum is broad. In this study, we aim to contribute to the understanding of the continuing expansion of the SCN1A-related disease phenotypes and the correlation between the type of the gene alterations and the resulting phenotypic spectrum.

\section{Material and Methods}

SCN1A gene alterations were detected in 10 patients who were suspected to have SMEI, SMEB and/or unspecific epileptic encephalopathy between 2013 and 2016. Clinical data including the age at first seizure, seizure types, presence of prolonged seizures, precipitating factors such as vaccination or fever, response to AEDs, developmental course, family history of febrile or afebrile seizures, electroencephalogram (EEG) and neuroimaging findings were recorded. Epileptic seizures of the patients were described according to the criteria of the Commission on Classification and Terminology of ILAE 2010. ${ }^{14}$ The diagnosis of SMEI was made according to the following criteria mentioned in the original report of Dravet et al. ${ }^{15}$ (1) high incidence of family history of epilepsy or febrile convulsions; (2) normal physical and neurologic development before onset; (3) appearance of seizures during the first year of life in the form of generalized or unilateral febrile and afebrile clonic seizures, secondary appearance of myoclonic jerks, and often partial seizures; (4) no paroxysmal discharge on the EEG in the early stages, but possible subsequent appearance of generalized spike-waves, polyspike-waves, and focal abnormalities; with possible early appearance of photosensitivity (5) normal psychomotor development initially, but retardation becomes evident from the second year of life, together with the appearance of ataxia, pyramidal signs, and interictal myoclonus; and (6) all seizure types are resistant to every form of treatment. A diagnosis of SMEI was established only if all these six criteria were fulfilled. Patients whose features are almost identical to those of SMEI but did not fully consistent with the accepted diagnostic criteria of SMEI are identified as having SMEI-borderline (SMEB). ${ }^{8}$

Genomic DNA from the peripheral blood lymphocytes of all individuals were extracted with QIAamp DNA Blood Mini Kit (Qiagen GMBH, Hilden, Germany) using standard procedures. All coding exons and exon-intron boundaries of SCN1A gene were amplified by polymerase chain reaction (PCR) using Helix Amp TM Ready-2X-Multiplex version 2.0 PCR mix (NanoHelix). Purified PCR products were Sanger sequenced and each variation was confirmed by repeated PCR and resequencing. Variation descriptions were done according to the nomenclature recommended by the Human Genomic Variation Society (HGVS). All variations were checked from mutation and SNP databases (Human Genome Mutation Database-HGMD, National Center for Biotechnology Information-NCBI/SNP, ensembl. org). Furthermore, in silico programs, such as SIFT, PolyPhen2, Mutation Taster, KD4V, were used for describe the pathogenicity of novel variations in coding exons and exon-intron boundaries. All participants provided written informed consent for participation in the study, which was approved by the Institutional Ethics Committee (Ethical approval number: 2016-23-14).

\section{Results}

The clinical features, EEG and neuroimaging findings and characteristics of SCN1A gene alterations of the study patients are shown in Tables I, II. Of 10 patients who had SCN1A gene alteration, 5 had SMEI phenotype with the classical presentation. The mean onset of seizures was between 4 and 6 months of age. The patient's clinical presentations did not differ from known SMEI phenotypes in regard to seizure characteristics, EEG findings, AED resistance and subsequent psychomotor retardation. Of these patients, 4 had formerly known SCN1A mutations (patients 5 and 8 had nonsense mutations, patients 6 and 9 had splice acceptor mutations). But, patient-7 was 


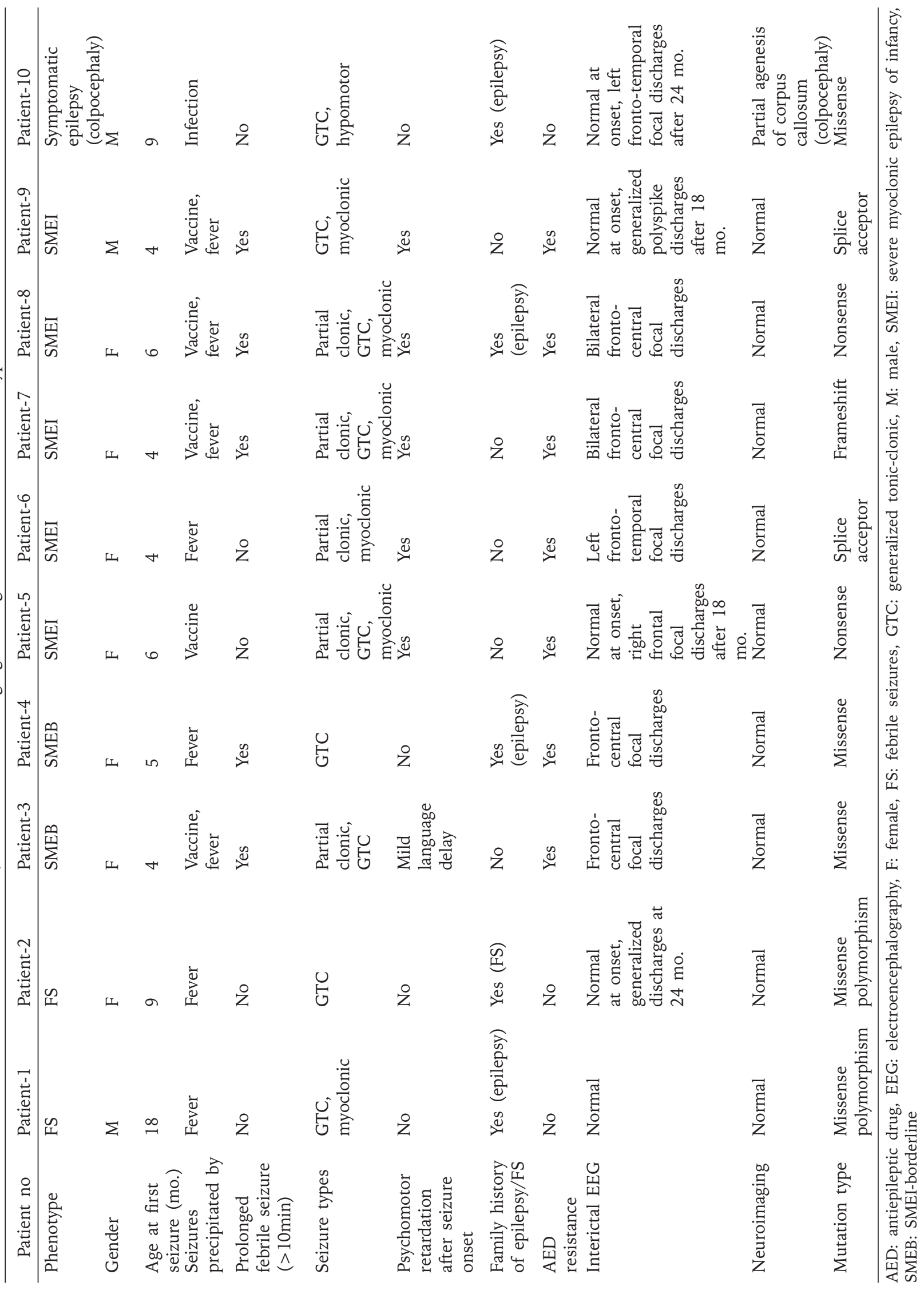




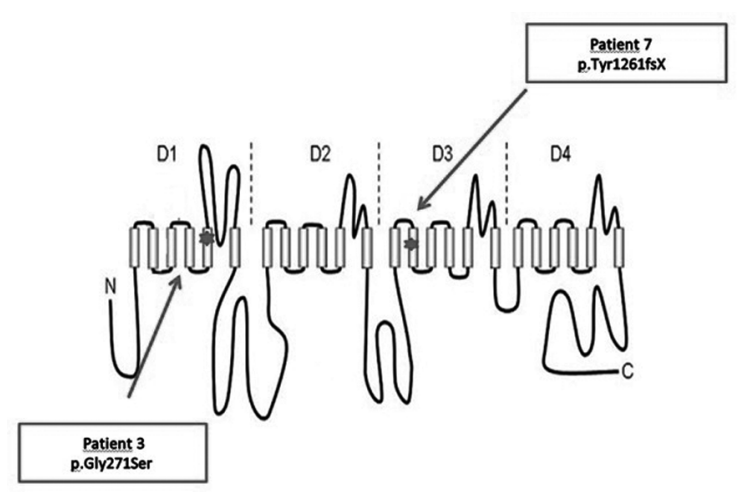

Fig. 1. Locations of the novel SCN1A mutations of patient-3 and patient-7.

found a novel frameshift mutation not defined previously (Fig. 1).

Two patients had SMEB phenotype. The seizure type was focal clonic in patient-3 and generalized tonic-clonic in patient-4. The seizures were drug-resistant in both. None of these cases had myoclonic seizures. At the last visit, patient- 3 was 4 years-old and had mild language delay and patient- 4 was 6 years-old and showed mild concentration and attention deficits without psychomotor delay. Concerning the types of mutations, patient 3 had a novel missense mutation. The mutation of patient- 4 was a previously defined mutation.

Another 2 patients with SCN1A variation presented with recurrent simple febrile seizures. Patient-1 had 12 seizures between 18 and 50 months and patient- 2 had 10 seizures between 9 and 48 months of age. Seizure types were substantially generalized tonic-clonic. But patient-1 displayed eyelid myoclonus in addition to generalized tonic-clonic seizures after 3 years of age. These two patients did not have any seizures beyond 4 years of age. Patient- 1 was 6 years-old and patient- 2 was 5 years-old at their last visits showing normal psychomotor development. These two patients were found to have the same missense variation which were previously described as polymorphism in various databases.

Among the study cases, one patient was diagnosed with symptomatic epilepsy (patient-10). Between 9 months and 4 years of age, he experienced 6 afebrile seizures, which all occurred during upper respiratory tract infections. The seizure types were focal clonic and generalized tonic-clonic. He did not have myoclonic seizures. He had mild psychomotor retardation. His magnetic resonance imaging showed hypoplasia of corpus callosum and colpocephaly. His genetic investigation showed a previously described missense mutation.

\section{Discussion}

The relationship between genotype and phenotype is obscure in SCN1A mutations. However, there are reports indicating some correlation. It has been reported that, mild generalized epilepsy and FS had the highest frequency of missense mutations that do not result in gross protein malformations. For more severe phenotypes, including SMEI and

Table II. Characteristics of SCN1A Gene Alterations of the Patients.

\begin{tabular}{lllllll}
\hline $\begin{array}{l}\text { Patient } \\
\text { No. }\end{array}$ & cDNA & Protein & Type & Exon & Topology & Phenotype \\
\hline 1 & $3199 \mathrm{G}>\mathrm{A}$ & Ala1067Thr & Missense & 16 & DII-DIII & FS (polymorphism) \\
2 & $3199 \mathrm{G}>\mathrm{A}$ & Ala1067Thr & Missense & 16 & DII-DIII & FS (polymorphism) \\
3 & $811 \mathrm{G}>\mathrm{A}$ & Gly271Ser & Missense & 6 & DI/S5 & SMEB (novel) \\
4 & $243 \mathrm{C}>\mathrm{A}$ & Asp81Glu & Missense & 1 & N-Terminal & SMEB \\
5 & $664 \mathrm{C}>\mathrm{T}$ & Arg222X & Nonsense & 5 & DI/S4 & SMEI \\
6 & $3706-$ & - & Splice acceptor & IVS18 & DIII/S1 & SMEI \\
7 & 1G $>$ A & & & & & \\
8 & $3783 \mathrm{delC}$ & Tyr1261fsX & Frameshift & 19 & DIII/S2 & SMEI (novel) \\
9 & $3615 \mathrm{G}>\mathrm{A}$ & Trp1205X & Nonsense & 18 & DII-DIII & SMEI \\
& 4582- & - & Splice acceptor & IVS24 & DIII-DIV & SMEI \\
10 & $1625 \mathrm{~T}>\mathrm{A}$ & Arg542Gln & Missense & 10 & & \\
\hline
\end{tabular}

FS: febrile seizures: SMEI: severe myoclonic epilepsy of infancy, SMEB: SMEI-borderline 
SMEB, the rate of missense mutations is decreasing. Missense mutations identified in severe phenotypes occurred more frequently in the pore region of $\mathrm{Na}_{\mathrm{v}} 1.1$ than those underlying milder phenotypes. ${ }^{16}$ Seventy to eighty percent of children with SMEI have point mutations or gross rearrangements in the SCN1A gene. ${ }^{17}$ Loss of function was postulated as the mechanism underlying truncating mutations, whereas missense mutations may involve more complex mechanisms and are possibly affected by other modifier genes or environmental factors. ${ }^{18} \mathrm{~A}$ total of $40-50 \%$ of mutations associated with the SMEI phenotype are missense in nature with 50-60\% truncating, whereas almost all the mutations associated with GEFS-phenotypes are missense. ${ }^{4}$ It is not well-understood why certain missense mutations are linked to severe phenotypes and others are not. Controversies remain as to whether missense mutations are clustered in specific regions of the SCN1A protein or whether they occur randomly across ion transport sequences, regions sharing significant homology with other ion channels and transporters. ${ }^{19}$ In our study, missense polymorphism was detected in 2 patients with recurrent FS. Of the remaining patients, missense mutations were detected in $3(37.5 \%)$, truncating mutation (one frameshift, two nonsense) in 3 (37.5\%) and splicing acceptor mutation in 2 patients $(25.0 \%)$. In 3 patients with missense mutation, 2 had clinical findings compatible with SMEB, and the other had symptomatic epilepsy. Furthermore, the patients with splicing acceptor and truncating mutation had clinical findings which were associated with SMEI. It has been reported that heterozygous loss-of-function mutations, such as truncations or the other mutations in the S5 and S6 regions which constitute the pore region would result in reduced channel density, and this may be the common underlying effect of all SMEI mutations. ${ }^{20}$ Also, missense mutations in cases with severe phenotype are found to be clustered in the voltage sensor region. ${ }^{21}$ In the present study, patient 5 , who had clinical features of SMEI, had nonsense mutation which was detected in the S4 transmembrane segment (voltage sensor region). Also, a missense mutation was detected in S5 segment of the sodium channel pore region in patient 3 who had SMEB phenotype. The mutations of this region are usually associated with severe clinical events and may also be related to atypical SMEI and GEFS + as reported in previous literature. The fact that similar mutations cause two different phenotypes implies that other environmental or genetic factors are possibly associated with SMEI. ${ }^{22}$

Novel mutations were detected in two of our patients. One of them, patient-3, had p.Gly271Ser mutation which was located in DIS5 segment of SCN1A gene. Previously, a missense mutation (p.Gly265Trp) which was also located in the same region was reported in a patient who had clinical findings associated with SMEI. But, the clinical findings of our patient were not typical for SMEI. At her last visit at 4-year-old, she had only mild language delay, but no motor retardation. The clinical features of the other patient (patient-7) were associated with SMEI phenotype and this novel mutation (p.Tyr1261fsX) was located in DIIIS2 region. In the same region, p.Leu1269fsX mutation was reported previously, which was also associated with SMEI and coexisting severe mental retardation and ataxia. ${ }^{23}$

Patients with a mutation, either truncating or missense, on linker regions had significantly later onset of disease. ${ }^{24}$ In our current study, mutation location involved linker regions in 3 patients. Two of them (patient-8 and patient-9) had clinical features of SMEI whereas patient-10 who had mutation p.Arg542Gln in DI-DII region, suffered symptomatic epilepsy. This previously reported variant was associated with juvenile myoclonic epilepsy, autism spectrum disorder, GEFS + and infantile epilepsy with variable penetrance. In a recent study, the authors reported that p.Arg542Gln mutation is not a pathogenic variant, based on the original reports, the high frequency in control individuals and missing or negative segregation and functional result. ${ }^{25}$ But the same mutation was reported in another study in a 5 year-old girl who was operated due to cleft palate and also suffered absence and atonic seizures. In this study, the authors suggested that the mutation disrupts the predicted tyrosine kinase site and destroys channel function. This may have a regulatory effect on channel activity and cause a milder phenotype. ${ }^{24}$ Our patient mostly suffered generalized tonic-clonic and focal clonic seizures. MRI demonstrated thinning of the corpus callosum and colpocephalic 
appearance of lateral ventricules. This finding is not reported previously.

In conclusion, our results show that SCN1A mutation spectrum continues to expand. The genetic and clinical data accumulated for SCN1A-related disorders will help to better understand and update the knowledge on pathophysiological mechanisms and genotypephenotype correlations.

\section{REFERENCES}

1. Escayg A, MacDonald BT, Meisler MH, et al. Mutations of SCN1A, encoding a neuronal sodium channel, in two families with GEFS+. Nat Genet 2000; 24: 343345 .

2. Plummer NW, Meisler MH. Evolution and diversity of mammalian sodium channel genes. Genomics 1999; 57: 323-331.

3. Gao QW, Hua L, Wang J, et al. A point mutation in SCN1A 5' genomic region decreases the promoter activity and is associated with mild epilepsy and seizure aggravation induced by antiepileptic drug. Mol Neurobiol 2017; 54: 2428-2434

4. Claes LR, Deprez L, Suls A, et al. The SCN1A variant database: a novel research and diagnostic tool. Hum Mutat 2009; 30: E904 -E920.

5. Dravet C. Les epilepsies garaves de l'enfant. Vie Med 1978; 8: 543-548.

6. Dravet C, Roger J, Bureau M, et al. Myoclonic epilepsies in childhood. In: Akimoto $\mathrm{H}$, Kazamatsuri H, Seino M, et al. (eds). Advances in Epileptology: the XIII Epilepsy International Symposium. New York: Raven Press, 1982: 135-140.

7. Oguni H, Hayashi K, Awaya Y, et al. Severe myoclonic epilepsy in infants: a review based on the Tokyo Women's Medical University series of 84 cases. Brain Dev 2001; 23: 736-748.

8. Fukuma G, Oguni H, Shirasaka Y, et al. Mutations of neuronal voltage-gated $\mathrm{Na}+$ channel $\alpha 1$ subunit gene SCN1A in core severe myoclonic epilepsy in infancy (SMEI) and in borderline SMEI (SMEB). Epilepsia 2004; 45: 140-148.

9. Wallace RH, Scheffer IE, Barnett S, et al. Neuronal sodium-channel alpha1-subunit mutations in generalized epilepsy with febrile seizures plus. Am J Hum Genet 2001; 68: 859-865.

10. Singh R, Scheffer IE, Crossland K, Berkovic SF. Generalized epilepsy with febrile seizures plus: A common childhood-onset genetic epilepsy syndrome. Ann Neurol 1999; 45: 75-81.

11. Fujiwara T, Sugawara T, Mazaki-Miyazaki E, et al. Mutations of sodium channel alpha subunit type 1 (SCN1A) in intractable childhood epilepsies with frequent generalized tonic-clonic seizures. Brain 2003; 126: $531-546$
12. Wallace RH, Hodgson BL, Grinton BE, et al. Sodium channel alpha1-subunit mutations in severe myoclonic epilepsy of infancy and infantile spasms. Neurology 2003; 61: 765-769.

13. Mantegazza M, Gambardella A, Rusconi R, et al Identification of an Nav1.1 sodium channel (SCN1A) loss-of-function mutation associated with familial simple febrile seizures. Proc Natl Acad Sci U S A 2005; 102: 18177-18182.

14. Berg AT, Berkovic SF, Brodie MJ, et al. Revised terminology and concepts for organization of seizures and epilepsies: Report of the ILAE Commission on Classification and Terminology, 2005-2009. Epilepsia 2010; 51: 676-685.

15. Commission on Classification and Terminology of the ILAE. Proposal for revised classification of epilepsies and epileptic syndromes. Epilepsia 1989; 30: 389-399.

16. Meng $\mathrm{H}, \mathrm{Xu} \mathrm{H}$, Yu L, et al. The SCN1A mutation database: Updating information and analysis of the relationships among genotype, functional alteration, and phenotype. Hum Mutat 2015; 36: 573-580.

17. Depienne C, Trouillard O, Saint-Martin C, et al. Spectrum of SCN1A gene mutations associated with Dravet syndrome: Analysis of 333 patients. J Med Genet 2009; 46: 183-191.

18. Escayg A, Goldin AL. Sodium channel SCN1A and epilepsy: Mutations and mechanisms. Epilepsia 2010, 51: $1650-1658$

19. Kanai K, Yoshida S, Hirose S, et al. Physicochemical property changes of amino acid residues that accompany missense mutations in SCN1A affect epilepsy phenotype severity. J Med Genet 2009; 46: 671-679.

20. Wallace R. A plethora of SCN1A mutations: What can they tell us? Epilepsy Currents 2005; 5: 17-20.

21. Kanai K, Hirose S, Oguni H, et al. Effect of localization of missense mutations in SCN1A on epilepsy phenotype severity. Neurology 2004; 63: 329-334.

22. Lossin C, Rhodes TH, Desai RR, et al. Epilepsyassociated dysfunction in the voltage-gated neuronal sodium channel SCN1A. J Neurosci 2003; 23: 11289 11295.

23. Zucca C, Redaelli F, Epifanio R, et al. Cryptogenic epileptic syndromes related to SCN1A: Twelve novel mutations identified. Arch Neurol 2008; 65: 489-494.

24. Usluer S, Salar S, Arslan M, et al. SCN1A gene sequencing in 46 Turkish epilepsy patients disclosed 12 novel mutations. Seizure 2016; 39: 34-43.

25. Lal D, Reinthaler EM, Dejanovic B, et al. Evaluation of presumably disease causing SCN1A variants in a cohort of common epilepsy syndromes. PLoS One 2016; 11: e0150426. 